\title{
One Dimensional Wavelet Transforms and their Application to T-ray Pulsed Signal Identification
}

\author{
Xiaoxia Yin ${ }^{a}$ and Brian W.-H. $\mathrm{Ng}^{a}$ and Bradley Ferguson ${ }^{a b}$ and \\ Samuel P. Mickan ${ }^{a}$ and Derek Abbott ${ }^{a}$ \\ ${ }^{a}$ Centre for Biomedical Engineering and School of Electrical \& Electronic Engineering, The \\ University of Adelaide, SA 5005, Australia; \\ ${ }^{b}$ Tenix - Electronic Systems Division 2nd Avenue, Technology Park, Mawson Lakes, SA 5095, \\ Australia
}

\begin{abstract}
This study investigates the application of one dimensional discrete wavelet transforms in the classification of T-ray pulsed signals. The Fast Fourier Transform (FFT) is used as a feature extraction tool and a Mahalanobis distance classifier is employed for classification. In this work, soft threshold wavelet shrinkage de-noising plays an important part in de-noising and reconstructing T-ray pulsed signals.

In addition, Mallat's pyramid algorithm and a local modulus maxima method to reconstruct T-ray signals are investigated. Particularly the local modulus maxima method is analyzed and comparisons are made before and after reconstruction of signals. The results demonstrate that these two methods are especially effective in analyzing and reconstructing T-ray pulsed responses.

Moreover, to test wavelet de-noising effectiveness, the accuracy of the classification is calculated and results are displayed in the form of scatter-plots. Results show that soft threshold wavelet shrinkage de-noising improves the classification accuracy and successfully generates visually pleasing scatter plots at selected three frequency components.
\end{abstract}

Keywords: T-ray, Discrete Wavelet Transform, Wavelet Shrinkage de-noising, Stein's Unbias Risk Estimator, wavelet maxima

\section{INTRODUCTION}

T-rays have wavelengths in the submillimetre range from $30 \mu \mathrm{m}$ to $3000 \mu \mathrm{m}$, corresponding to the frequency range from $0.1 \mathrm{THz}$ to $10 \mathrm{THz}$ in the electromagnetic spectrum. T-rays have promising potential in both in in vivo and in vitro biosensing owing to (i) their non-invasive property, and (ii) the fact that biomolecules have rich resonances in the $\mathrm{THz}$ region. The ultimate aim of our work is to perform automatic classification of data obtained from T-ray tomography. This requires sophisticated signal processing techniques, which are capable of analyzing and dealing with measured image statistics and automatically identifying materials. T-ray tomographic classification is a technique that reconstructs and classifies geometrical images with multiple layers, based on an advanced coherent detection method. This paper investigates three methods of reconstructing and classifying T-ray pulsed signals. T-ray computed tomography data, in the form of T-ray pulsed signals, are analyzed after performing a 1D discrete wavelet transform (DWT) - a useful mathematical tool in time-frequency analysis, which allows excellent local characterization of signals. ${ }^{1,2}$ It has been successfully applied in a number of areas such as imaging de-noising, reconstruction, and feature extraction. ${ }^{3}$

Discrete wavelet transforms can be efficiently employed to process and reconstruct transient signals, ${ }^{4}$ such as a T-ray pulsed response, because of their similarity in shape to wavelets. ${ }^{5}$ Exploitation of wavelet shrinkage for denoising is implemented using soft thresholding based on the heuristic SURE (Stein's Unbiased Risk Estimates) method - this is shown to improve classification performance. Mallat's pyramid algorithm is viewed as a critical algorithm for the calculation of DWT coefficients and reconstruction of T-ray signals. ${ }^{4}$ Based on the importance

Further author information: (Send correspondence to Derek Abbott)

Derek Abbott: E-mail: dabbott@eleceng.adelaide.edu.au, Telephone: +61-8-8303-5748

Photonics: Design, Technology, and Packaging II, edited by Derek Abbott, Yuri S. Kivshar, Halina H. Rubinsztein-Dunlop, Shanhui Fan, Proc. of SPIE Vol. 6038, 603829, (2006) $\cdot$ 0277-786X/06/\$15 · doi: 10.1117/12.673530 
of analyzing signal, a local modulus maxima reconstructed curve is demonstrated, which is well adapted to the T-ray responses.

This paper looks at the application of DWT in reconstructing and classifying T-ray signals.

\section{THE METHODOLOGY}

\subsection{Realization of DWT of T-ray Pulsed Responses}

T-ray pulses are continuous signals that are sampled before being analysed with a Discrete Wavelet Transform (DWT), has translation- and scale steps. ${ }^{6}$ The DWT of a T-ray pulsed response is computed by correlating an input discrete T-ray pulsed response to a function, such as the Daubechies wavelet, called a "mother wavelet". This process is achieved by moving the wavelet signal along the input T-ray pulsed signals, and also changing the scale (that is, expanding or contracting the mother wavelet). As a result, a representation of the original T-ray pulsed signals consisting of different scales or resolutions at different times (positions) is achieved.

\subsection{De-noising, Reconstruction and Classification Using Heuristic SURE Soft Threshold}

Wavelets are a mathematical tool that enable powerful real-time analysis of non-stationary signals, such as T-ray pulse signals. Such transient signals cannot be predicted at different times ${ }^{7}$ and cannot be analyzed and handled as stationary signals. An important preprocessing stage for non-stationary signals is to remove unwanted noise present in the captured signal. Wavelet shrinkage de-noising is proposed for this purpose. It achieves good de-noising performance through a reduction in the number non-zero wavelet coefficients required to represent the T-ray pulse signals. ${ }^{8}$ Wavelet shrinkage de-noising attempts to remove noise across all the frequency sub-bands at all times, ${ }^{9}$ something obviously not possible with adaptive noise-removing filters.

\section{A Brief Introduction to Soft Threshold Shrinkage De-noising}

There are several sources of noise in modern T-ray systems. These are caused by both systematic and random errors in the systems. The noise of T-ray signals includes the T-ray emitter noise, Johnson and shot noise from the T-ray detector as well as the thermal background radiation. ${ }^{10,11}$

Soft threshold wavelet de-noising is known to be effective in de-noising the T-ray pulsed response and yielding visually satisfying signals. The operation of soft threshold, with threshold $\lambda$, is described using the following equation:

$$
D(U, \lambda)=\operatorname{sgn}(U) \max (0,|U|-\lambda) .
$$

As the abosolute value of the soft threshold shrinkage coefficients are above the threshold, ${ }^{12}$ a soft threshold has better continuity and makes algorithms mathematically controlled, ${ }^{13}$ as well as effectively shrinking pure noise coefficients.

There are three steps involved in achieving a non-linear soft threshold:

(1) Apply wavelet decomposition techniques to realize a forward wavelet transform.

(2) Realize non-linear shrinkage de-noising by applying Eq. (1) to the detail coefficients. A threshold is selected for each wavelet transform level from 1 to 3.

(3) Compute the inverse wavelet transform to reconstruct the de-noised T-ray pulsed signals. Compute IDWT based $^{14}$ on the original level 3 approximation coefficients and the modified detail coefficients of from levels 1 to 3.

\section{What is SURE?}

The nonlinear shrinking of coefficients in the wavelet transform domain is a nonparametric method. Here, the threshold of heuristic Stein's Unbiased Risk Estimates (SURE) ${ }^{12}$ is selected, which uses a local threshold and is estimated adaptively for each level $L$. The calculation of SURE is described briefly as follows. 
Let vector $\mu=\left(\mu_{i}: i=1, \ldots d\right)$, where the length is $d$. Let $\mathbf{x}=x_{i}$ with similar distributions to $N\left(u_{i}, 1\right)$. SURE is used to estimate the unbiased risk $E\left\|\widehat{u}_{i}^{(t)}(\mathbf{x})-\mu\right\|^{2}$, which means the estimate of energy loss that is obtained based on Stein's result ${ }^{15}$ :

$$
\operatorname{SURE}(\lambda ; \mathbf{x})=d-2 \cdot \#\left\{i:\left|x_{i}\right|<\lambda\right\}+\sum_{i=1}^{d} \min \left(\left|x_{i}\right|, \lambda\right)^{2}
$$

where $\widehat{u}$ is the soft threshold estimator $\widehat{u}_{i}^{(\lambda)}(\mathbf{x})=\eta_{\lambda}\left(x_{i}\right)$. Vector $\mathbf{x}$ contains noisy wavelet coefficients in a subband and the aim of experiment is to calculate the threshold $\lambda$ that minimizes $\operatorname{SURE}(\lambda ; \mathbf{x}){ }^{12}$ Here, \# means the number of the coefficients which are smaller than the threshold of noisy signals .

\section{What is Heuristic SURE?}

Wavelet shrinkage de-noising in this investigation uses heuristic SURE soft threshold, which is selected and determined based on the underlying model

$$
X(\tau)=S(\tau)+N(\tau)
$$

where $S(\tau)$ is a real-valued T-ray pulsed signal with additive system noise $N(\tau)$, both of which are functions of time $\tau$ and are sampled. There are two options for determining the heuristic SURE soft threshold.

First, 'heursure' is used for the soft threshold estimation, and the threshold is selected based on Stein's Unbiased Estimate of Risk (quadratic loss function). A particular threshold value $\lambda$ is calculated to estimate the risk. The threshold value is the minimum of the risks in $\lambda$.

Second, if the signal-to-noise ratio is very small, the SURE estimate is very noisy. In this situation, minimax performance is yielded by a fixed form threshold, which is multiplied by a small factor proportional to $\log ($ length $(\mathbf{x}))$.

\section{Classification}

To test the effectiveness after wavelet shrinkage de-noising, several samples of powders are classified and the features are acquired, after taking the FFT of reconstructed signals - three optimal frequency components are extracted by a Genetic Algorithm(GA). To demonstrate the effective classification after wavelet de-noising, visual scatter plots of amplitude at three optimum frequencies are produced.

The classification of the six kinds of powders and a control empty holder is carried out by a Mahalanobis distance classifier ${ }^{16}$ in Figure 5 . The Mahalanobis distance classifier is regarded as a simple and effective classifier. The classifier simply defines quadratic decision boundaries in the feature space to separate the classes. The Mahalanobis distance is defined as the distance from the mean value of the pointed class to a given point, where the given class is normalised by the different training vectors along their respective directions. ${ }^{16}$ For a given class, $i$, the distance is defined as,

$$
d_{i}(\mathbf{x})=\sqrt{\left(\mathbf{x}-\mathbf{u}_{\mathbf{i}}\right)^{T} \sum_{i}^{-1}\left(\mathbf{x}-\mathbf{u}_{\mathbf{i}}\right)} .
$$

The minimum Mahalanobis distance is then calculated to select the class of powders to obtain the final classification. In this experiment, a Mahalanobis distance classifier is trained using 3 frequency components on a separate set of powder data at thicknesses of $2 \mathrm{~mm}, 3 \mathrm{~mm}$ and $4 \mathrm{~mm}$, while the set of powder data at a thickness of $3 \mathrm{~mm}$ is used as test samples. Finally, the classification accuracy is represented by a confusion matrix for the classifier, which is demonstrated in Eq. 18. Additionally, the SNR of the denoised signal is measured by:

$$
\operatorname{SNR}(\mathrm{dB})=10 \log \left(\frac{\sum_{k=0}^{N-1} a(k)^{2}}{\sum_{k=0}^{N-1}[a(k)-b(k)]^{2}}\right),
$$

where $\mathbf{a}=[a(k)]$ is the raw T-ray signal and $\mathbf{b}=[b(k)]$ is the de-noised signal.

In this paper, the eighth order of Daubechies wavelet is employed to denoise T-ray signals with a transform depth of three levels and the corresponding performances are shown in Figure 2. Moreover, all the figures (except Figure 4), are produced by using a soda powder sample at a thickness of $3 \mathrm{~mm}$. 


\subsection{Perfect Reconstruction: Mallat's Pyramid Algorithm}

Mallat's pyramid algorithm is considered to hold a similar importance to FFT in analyzing signals. It is commonly used in the calculation of discrete wavelet transform coefficients. The Mallat's algorithm, in this experiment, is realized by a pair of mirror Finite Impulse Response (FIR) filters and the procedures of down sampling and up sampling, together with circular convolutions at each scale to calculate the approximate and detailed wavelet coefficients. The procedure of the experiment is as follows.

A T-ray pulsed signal, $f(t)$, can be represented as a sum of weighted scaling and wavelet functions:

$$
f(t)=\sum_{k} c_{j_{0}}(k) 2^{j_{0} / 2} \phi\left(2^{j_{0}} t-k\right)+\sum_{k} \sum_{j=1}^{j_{0}} d_{j}(k) 2^{j / 2} \psi\left(2^{j} t-k\right),
$$

$\phi(t)$ and $\psi(t)$ are the scaling and wavelet functions, respectively. In this paper, these functions, along with their translated and dilated versions, are assumed to form an orthogonal basis. ${ }^{4}$

The function of wavelet basis is defined as follows:

$$
\psi(t)=2^{-m / 2} \psi\left(2^{-m} t-n\right),
$$

where $\psi(t)$ belongs to space $V_{-1}$,

$$
\left\{\begin{array}{l}
\phi(t)=\sum_{n} h(n) \phi_{-1, n}(t)=\sqrt{2} \sum_{n} h(n) \phi(2 t-n) \\
\psi(t)=\sum_{n} g(n) \phi_{-1, n}(t)=\sqrt{2} \sum_{n} g(n) \phi(2 t-n) .
\end{array}\right.
$$

The coefficients of a pair of mirror FIR filters have such a relationship as:

$$
g(n)=(-1)^{n} h(1-n),
$$

where $h(n)$ and $g(n)$ are the coefficients of the low-pass and high-pass filters, respetively. T-ray pulsed responses are capable of being approximated by smooth projection onto scaling subspaces and detailed projection onto the subspaces of a wavelet.

Wavelet coefficients are calculated based on the fast Mallat's algorithm, utilising the low-pass and high-pass filters. Project an arbitrary $f(t) \epsilon V_{j-1}$ to the spaces $V_{j}$ and $W_{j}$ to obtain the following formulae:

$$
\left\{\begin{array}{l}
c_{j+1, k}=\sum_{m} h(m-2 k) c_{j, m} \\
d_{j+1, k}=\sum_{m} g(m-2 k) c_{j, m}
\end{array}\right.
$$

where $c_{j+1, k}$ are the scaling coefficients corresponding to the space $V_{j+1, k}$ while $d_{j+1, k}$ are the wavelet coefficients corresponding to the space $W_{j+1}$. The filters corresponding to the Daubechies-8 wavelet are used in this paper. The equation of reconstruction of wavelet coefficients is,

$$
c_{j-1, m}=\sum_{k} c_{j, k} h(m-2 k)+\sum_{k} d_{j, k} h(m-2 k) .
$$

The processes of forward and inverse wavelet transforms are reversible, hence Perfect Reconstruction (PR) is built into the fabric of the DWT, subject to possible implementation issues such as bit-truncation errors.

\section{A Brief Introduction of the Calculation of Wavelet Maxima}

Wavelets are, by their nature, localized functions. Due to the repetition of rescaling, wavelets decompose a signal in details at different scales, thereby producing a multiresolution representation of data, which contain the all-important edges. ${ }^{17}$ As the resolution decreases dyadically when the sub-bands are generated, computational efficiency is achieved. ${ }^{18}$

The current experiment concentrates wavelet maxima reconstruction curve after 1D wavelet transform. The sharp variation of T-ray pulsed signals yields modulus maxima at different scales of $2^{j}$, which is detected by the 
first- and second-order derivatives. Let $\theta(x)$ represent a smooth function, and $\psi^{a}(x), \psi^{b}(x)$ be the first- and second-order derivatives of $\theta(x)$ and viewed as the wavelet functions. Therefore, there exist wavelet transforms at scale $s$ and position $x$,

$$
\begin{aligned}
& W_{s}^{a} f(x)=f * \psi^{a}(x) \\
& W_{s}^{b} f(x)=f * \psi^{b}(x)
\end{aligned}
$$

Where "*" represents the convolution operation. Based on the Eqs. 12 and 13, the following corresponding equations can be derived:

$$
\begin{gathered}
W_{s}^{a} f(x)=f *\left(s \frac{d \theta_{s}}{d x}\right)(x)=s \frac{d}{d x}\left(f * \theta_{s}\right)(x) \\
W_{s}^{b} f(x)=f *\left(s^{2} \frac{d^{2} \theta_{s}}{d x^{2}}\right)(x)=s^{2} \frac{d^{2}}{d x^{2}}\left(f * \theta_{s}\right)(x) .
\end{gathered}
$$

Let $W_{s}^{a} f(x), W_{s}^{b} f(x)$, and $f * \theta_{s}(x)$ denote the local extrema, the zeros cross and the inflection points. The sharp variation of the inflection points $f * \theta_{s}(x)$ is easily selected by the maxima of the absolute of first-order derivative And the position can be determined by the second-order derivative.

The function $f(x)$ can be reconstructed from dyadic wavelet transform with the summation:

$$
f(x)=\sum_{j=-\infty}^{+\infty} W_{2 j} f * \chi_{2 j}(x)
$$

and $\chi_{2 j}$ satisfies:

$$
\sum_{j=-\infty}^{+\infty} \hat{\psi}\left(2^{j} \omega\right) \hat{\chi}\left(2^{j} \omega\right)=1
$$

The difference ${ }^{3}$ between reconstructed signal $\tilde{f}$ and original signal is below $10^{-2}$ a.u.

\section{RESULTS AND DISCUSSION}

\subsection{Classification and reconstructed signals using wavelet shrinkage de-noising}

The option 'heursure' SURE soft threshold wavelet shrinkage de-noising is effective in dealing with T-ray pulsed noisy responses. A comparison of the T-ray noisy signals and de-noising T-ray signals are demonstrated in Figure 1. Figure 2 demonstrates the plots of wavelet coefficients before and after wavelet shrinkage de-noising of T-ray pulsed signals. Figure 3 shows the raw signal samples of the six different types of powders and their holder in the time domain.

Figure 4 shows amplitude scatter plots at 3 optimal frequencies obtained from a G.A.: $0.64 \mathrm{THz}, 0.79 \mathrm{THz}$ and $0.26 \mathrm{THz}$. In this figure, six different types of powders and their holders are separated clearly and classification is acquired and the accuracy of classification reaches $95 \%$ and is improved by $6 \%$ compared with the accuracy of raw measured T-ray pulsed signals.

To effectively show powder classification at three frequencies, the $3 \mathrm{D}$ scatter plots are reduced into $2 \mathrm{D}$ scatter plots. Three different possible combinations of $2 \mathrm{D}$ scatter plots have been made: amplitude at $0.79 \mathrm{THz}$ and 0.26 THz; amplitude at $0.64 \mathrm{THz}$ and $0.79 \mathrm{THz}$ together with amplitude at $0.26 \mathrm{THz}$ and $0.64 \mathrm{THz}$. The accuracy of classification is $92.72 \%, 87.96 \%, 87.96 \%$, respectively.

A confusion matrix for this classifier is shown in Eq. (17), which is used to illustrate the accuracy for the classification and describe the classifier performance. For each matrix element, $[i, j], i$ demonstrates the proportion of samples belonging to class $i$, and $j$ shows the proportion of the corresponding samples that were recognised as class $j$ because of the error, ${ }^{5}$ 


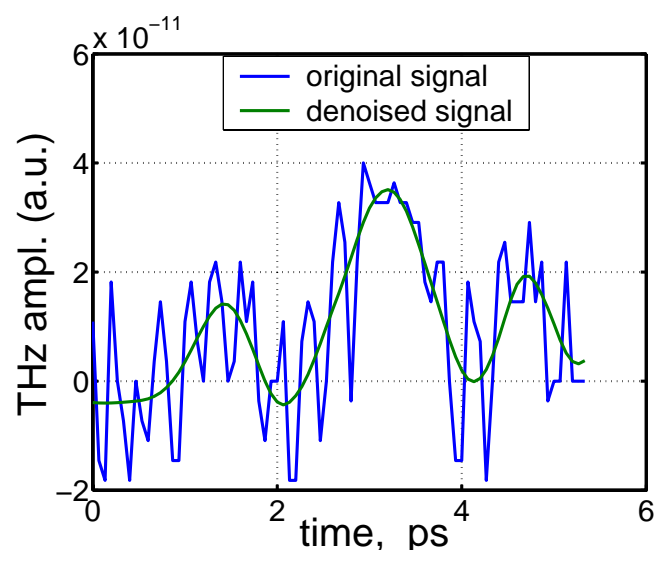

Figure 1. A comparison of the T-ray noisy signal and de-noising T-ray signal, which has a frequency cutoff at 4 THz.
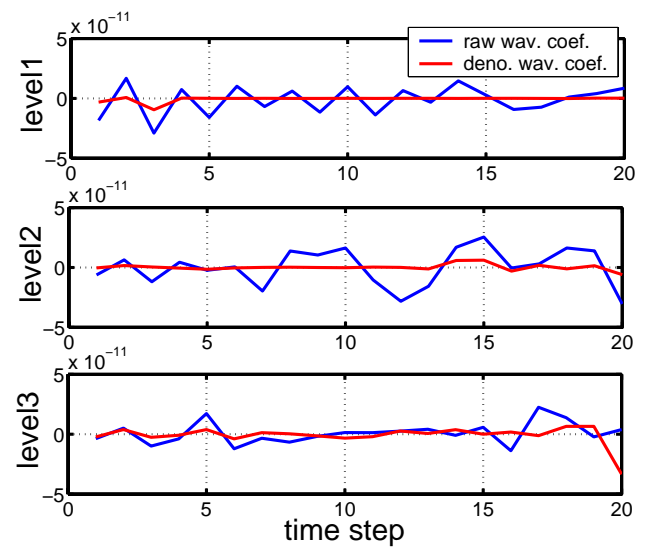

Figure 2. Detailed wavelet coefficient plots at 3 levels before and after wavelet shrinkage de-noising of T-ray pulsed signal. In order to display the change of wavelet coefficients, the half of time samples are kept, respectively. Each time sample equals to $0.0677 \mathrm{ps}$.

Proc. of SPIE Vol. 6038 603829-6 


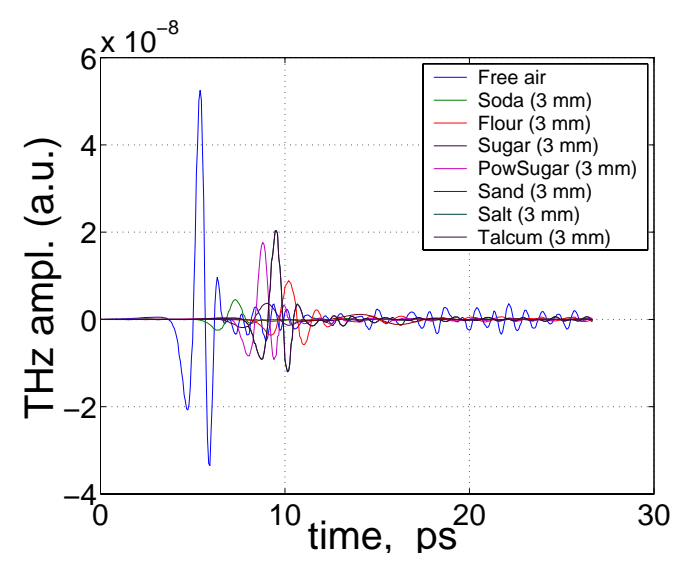

Figure 3. The raw T-ray pulsed signals after transmission through six different types of powders and their holder in the time domain. The six powders are compacted to $3 \mathrm{~mm}$ pellets in thickness each and are (i) wheat flour, (ii) baking soda, (iii) sucrose, (iv) powdered sucrose, (v) salt, and (vi) talcum powder. The seventh trace acts as control data and is obtained with an empty holder.

$$
\mathbf{S}=\left(\begin{array}{ccccccc}
1.0000 & 0 & 0 & 0 & 0 & 0 & 0 \\
0 & 0.7059 & 0 & 0 & 0 & 0 & 0 \\
0 & 0 & 1.0000 & 0 & 0 & 0 & 0 \\
0 & 0 & 0 & 1.0000 & 0 & 0 & 0 \\
0 & 0.2941 & 0 & 0 & 1.0000 & 0 & 0 \\
0 & 0 & 0 & 0 & 0 & 1.0000 & 0 \\
0 & 0 & 0 & 0 & 0 & 0 & 1.0000
\end{array}\right)
$$

After using soft threshold wavelet shrinkage denoising, the ratio of signal to noise (SNR) is $17 \mathrm{~dB}$. The classification accuracy at three frequencies is improved by at least $3 \%$ compared with the classification accuracy at 2 frequencies. Figure 4 shows one of these three graphs at frequency $0.79 \mathrm{THz}$ and $0.64 \mathrm{THz}$.

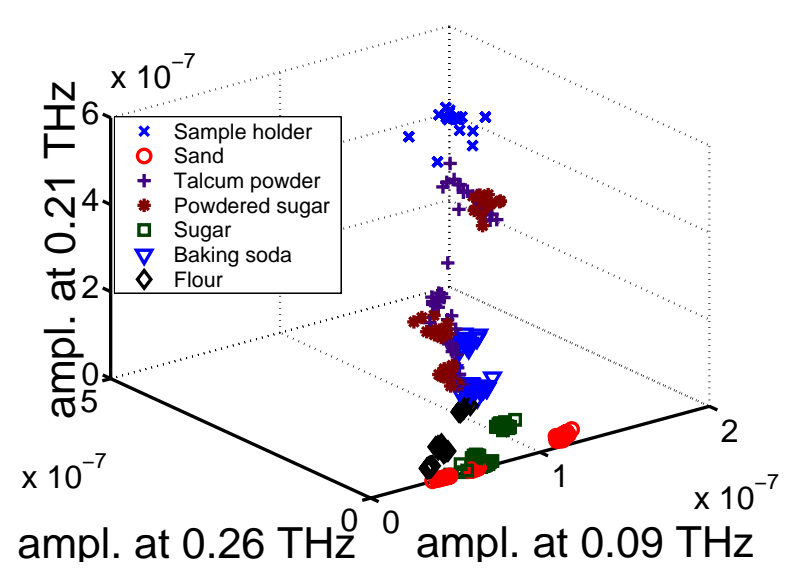

(a)

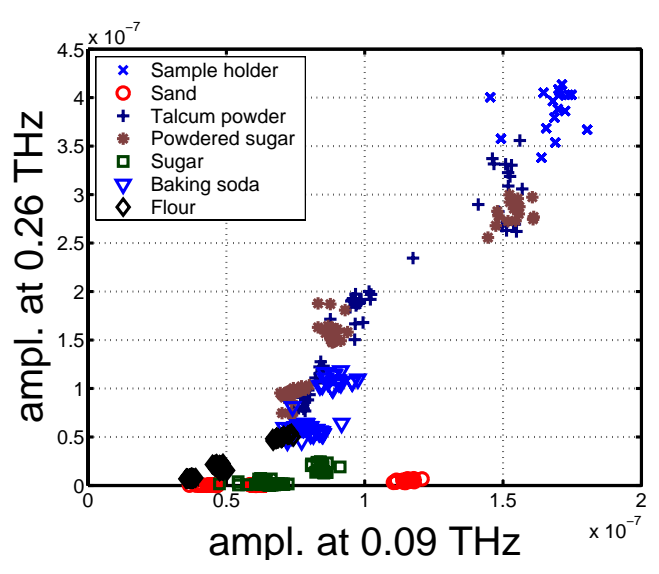

(b)

Figure 4. Amplitude scatter plots. (a) Amplitude scatter plot at 3 optimal frequencies: $0.64 \mathrm{THz}, 0.79 \mathrm{THz}$ and 0.26 THz. (b) Amplitude scatter plot at two frequencies: $0.79 \mathrm{THz}$ and $0.64 \mathrm{THz}$. 


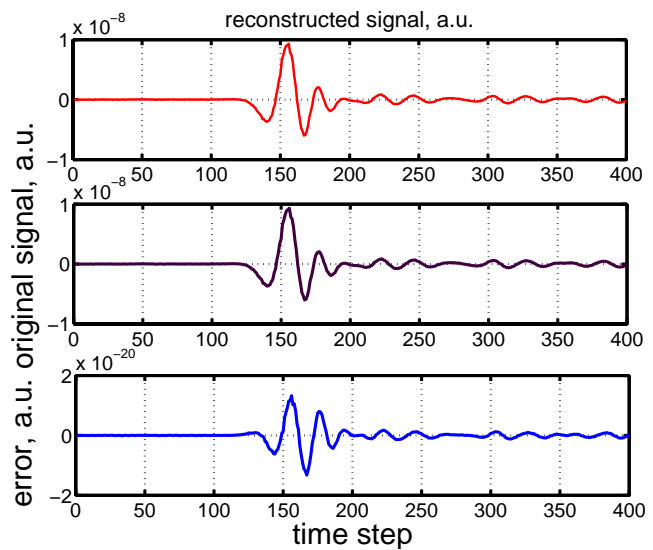

Figure 5. The reconstructed and original signals as well as the difference between these two signals using Mallat's algorithm.

All these data and the results indicate that DWT is extremely effective in analyzing T-ray signal and plays a critical role in achieving T-ray signal classification. In particularly, wavelet de-noising and reconstruction using heuristic SURE soft threshold is capable of achieving quite good scatter plots with better accuracy in the classification.

\subsection{Signal Reconstruction Using Mallat's Algorithm}

Applying Mallat's algorithm, the reconstructed and original signals along with the difference between these two signals are shown in Figure 5.

\subsection{Signal Reconstruction Using Local Modulus Maxima}

A plot of the position and the value of wavelet modulus of a pulsed signal together with the corresponding original signal of the soda sample at a thickness at $3 \mathrm{~mm}$ is demonstrated in Figure 8. The position and the value of the local modulus of the T-ray pulsed signal is shown quite clearly.

This experiment also shows that the T-ray pulsed signals can be perfectly reconstructed by wavelet maxima modelling with error less than $1 \%$, which is demonstrated in Fig. 6.

Figure 6 demonstrates the original T-ray pulsed signals with 400 time samples and reconstructed signal as well as the difference between these two signals. An emphasis is put on a stationary Wavelet Transform (SWT), which is adopted to decompose signals and calculate approximate and detailed wavelet coefficients without down and up sampling. Wavelet coefficient plots on scale and time planes are shown in Figure 7. The positions and the values of wavelet modulus are calculated and plotted at three different levels in Figure 8. The raw and reconstructed T-ray pulsed responses are shown at three levels in Figure 9.

Based on the above experimental results, the T-ray pulsed signals can be reconstructed with an error less than $1 \%$. Furthermore, this representation of reconstructed signals is well-adapted to the original one. It is a robust method to reconstruct and analyze T-ray pulsed signals using local modulus maxima.

\section{CONCLUSION}

In this investigation, a high classification accuracy is demonstrated by using DWT of T-ray pulsed responses. The results reveal that, a higher level of accuracy can be obtained after implementing heuristic SURE wavelet shrinkage de-noising. Reconstructed signals have good smoothness. The classification accuracy is high and scatter-plots are visually pleasing.

In addition, the local maxima technique allows the T-ray pulse responses to be reconstructed effectively from their wavelet coefficients. Many graphs including wavelet coefficient plots at different levels and scales and 


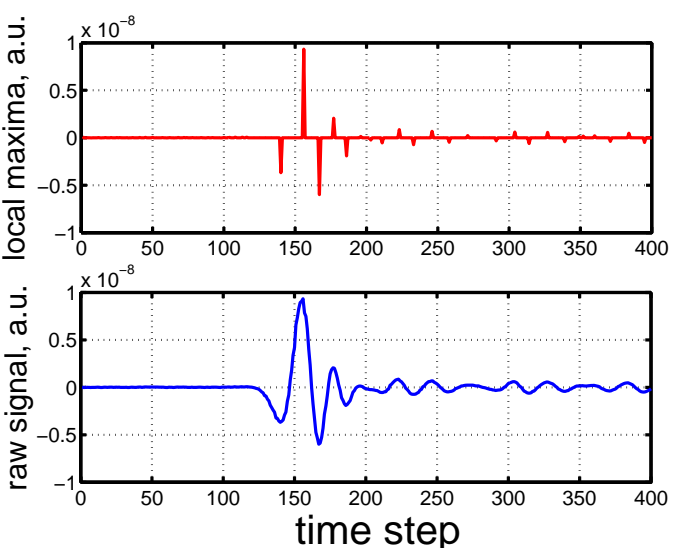

Figure 6. A plot of the position and the value of wavelet modulus of a pulsed signal. Baking soda powder at a thickness of $3 \mathrm{~mm}$ is the sample used here. Each time sample equals to $0.0677 \mathrm{ps}$.

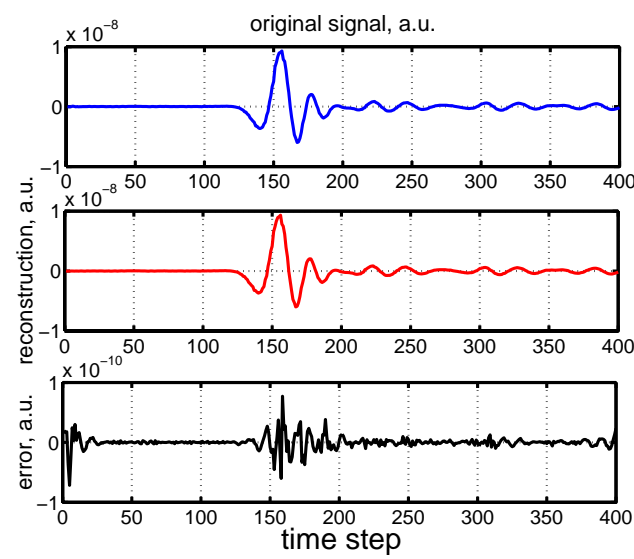

Figure 7. T-ray original signals and reconstructed signals implementing wavelet maxima and the their difference. Each time sample equals to $0.0677 \mathrm{ps}$.
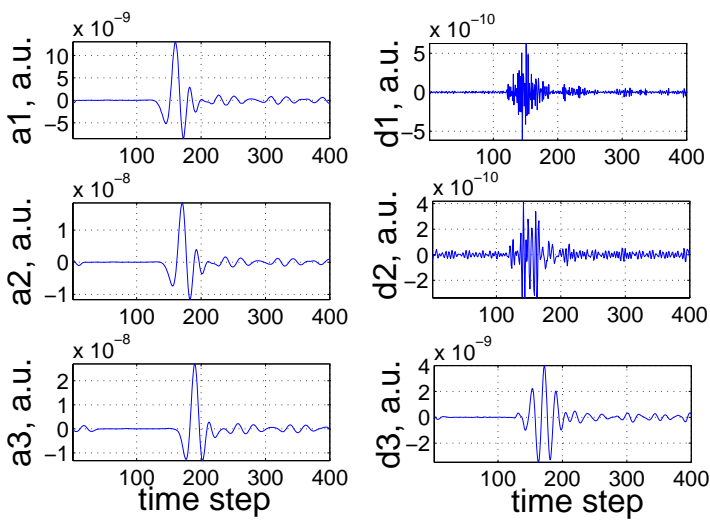

Figure 8. Approximate and detailed wavelet coefficient plots on scale and time planes. Each time sample equals to 0.0677 ps.

Proc. of SPIE Vol. 6038 603829-9 


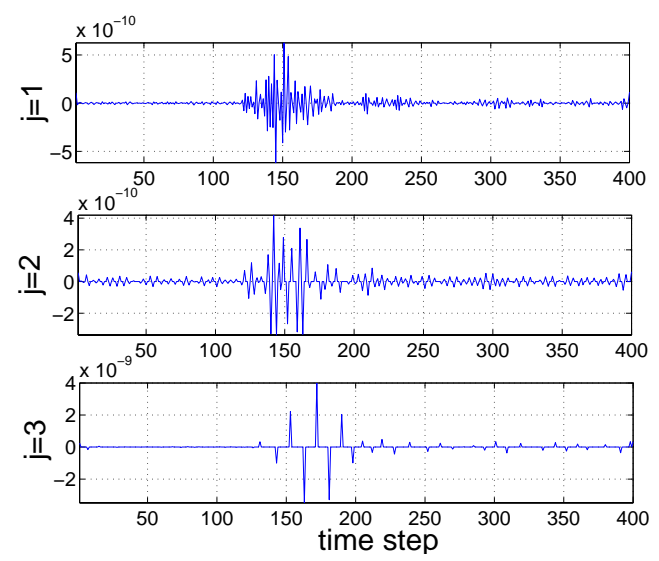

Figure 9. The position and the value of wavelet modulus are calculated and plotted at three different levels. Each time sample equals to 0.0677 ps.
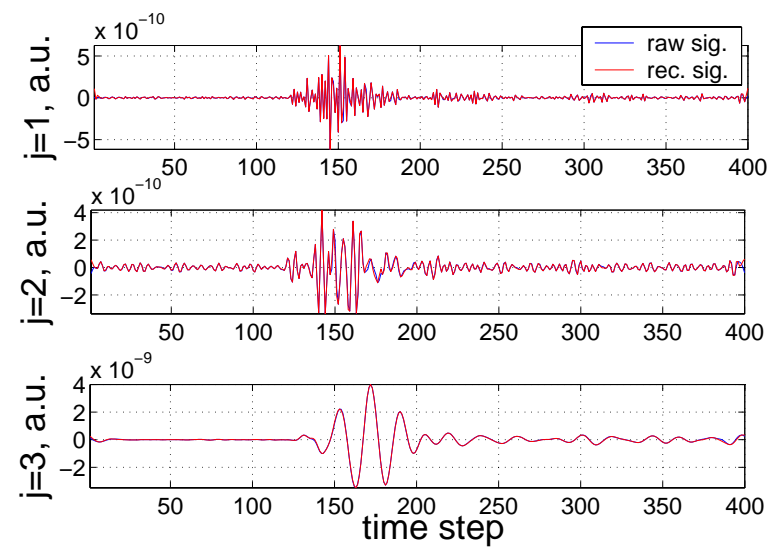

Figure 10. The raw and reconstructed T-ray pulsed responses at three levels. Each time sample equals to 0.0677 ps. 
reconstructed curves together with the difference between original and reconstructed signals have been presented. These results suggest that T-ray pulsed signals are capable of being effectively analyzed in the wavelet transform domain.

\section{REFERENCES}

1. S. G. Mallat, A Wavelet Tour of Signal Processing, 2nd edn, San Diego: Academic Press, CA, 1999.

2. Y. Tang, L. Yang, J. Liu, and H. Ma, Wavelet Theory and Its Application to Pattern Recognition, World Scientific, Singapore, 2000.

3. G. Qu, D. Zhang, and P. Yan, "Medical image fusion by wavelet transform modulus maxima," Optics Express 9(4), pp. 184-190, 2001.

4. Y. Guo, H. Zhang, X. Wang, and R. Cavallaro, "VLSI implementation of Mallat fast discrete wavelet transform algorithm with reduced complexity," IEEE Global Telecommunications Conference 1, pp. 25-29, 2001.

5. B. Ferguson, Three Dimensional T-ray Inspection Systems. PhD thesis, Electrical and Electronic Engineering, Adelaide University, 2004.

6. C. Valens, "A really friendly guide to wavelets, http://perso.wanadoo.fr/polyvalens/clemens/ wavelets.html\\#note1, (accessed: August 29 2005)."

7. S. Waddington and $\mathrm{S}$. Li, "Multiresolution signal analysis and wavelet decomposition, http://www. embedded.com/97/feat9607.htm, (accessed: August 29 2005)."

8. B. Ferguson and D. Abbott, "De-noising techniques for terahertz responses of biological samples," Elsevier Microelectronics Journal 32(12), pp. 943-953, 2001.

9. C. Taswell, "The what how and why of wavelet shrinkage denoising," IEEE Computer in Science and Engineering 2000, pp. 664-67, 2000.

10. A. Poppe, L. Xu, F. Krausz, and C. Spielmann, "Noise characterisation of sub-10-fs ti:sapphire oscillators I," IEEE Journal of Selected Topics in Quantum Electronics 4, pp. 179-184, 1998.

11. L. Duvillaret, F. Garet, and J. L. Coutaz, "Influence of noise on the characterization of materials by terahertz time-domain spectroscopy," The Optical Society of America B: Optical Physics 17, pp. 452-461, 2000.

12. R. Rangarajan, R. Venkataramanan, and S. Shah, "Imaging denoising using wavelet (wavelets and time frequency), http://bbs.matwav.com/index.jsp, (accessed: August 19 2005)."

13. D. L. Donoho, "De-noising by soft thresholding," IEEE Transactions on Information Theory 41(3), pp. 613627, 1995.

14. Mathworks, "Wavelet Toolbox, http://www.mathworks.com/access/helpdesk/help/toolbox/wavelet/ ch01\_i21.html, (accessed: August 16 2005)."

15. M. Johnstone and L. Donoho, "Adapting to smoothness via wavelet shrinkage," Journal of the Statistical Association 90(432), pp. 1220-1224, 1995.

16. J. Schürmann, Pattern Classification: Unified View of Statistical and Neural Approaches, John Wiley \& Sons, Inc., NewYork, 1996.

17. G. Strang and T. Nguyen, Wavelets and Filter Banks, Wellesley-Cambridge Press, Wellesley, MA, 1996.

18. S. Mallat, "A theory for multiresolution signal decomposition: the wavelet representation," IEEE Transactions on Pattern Analysis and Machine Intelligence, II(7), pp. 674-693, 1989. 